\title{
Letter to the Editor: Surveillance of $m c r-1$ and $m c r-2$ genes in Carbapenem-resistant Klebsiella pneumoniae strains from an Italian Hospital
}

C Venditti ${ }^{1}$, C Nisii ${ }^{1}$, S D’Arezzo ${ }^{1}$, A Vulcano ${ }^{1}$, A Di Caro ${ }^{1}$

1. National Institute for Infectious Diseases (INMI) L. Spallanzani - IRCCS, Rome, Italy

Correspondence: Antonino Di Caro (antonino.dicaro@inmi.it)

Citation style for this article:

Venditti C, Nisii C, D’Arezzo S, Vulcano A, Di Caro A. Letter to the Editor: Surveillance of mcr-1 and mcr-2 genes in Carbapenem-resistant Klebsiella pneumoniae strains from an Italian Hospital. Euro Surveill. 2017;22(35):pii=30604. DOI: http://dx.doi.org/10.2807/1560-7917.ES.2017.22.35.30604

Article submitted on 22 August 2017 / accepted on 30 August 2017 / published on 31 August 2017

To the editor: In a recent editorial [1], Kluytmans reports on the rise of plasmid-mediated colistin resistance that has occurred worldwide since its first description in 2016 [2]. Antimicrobial resistance, especially in Gram-negative bacteria, has been recognised as a serious threat to human health, and colistin, as well as tigecycline, is often the only therapeutic option for infections caused by carbapenem-resistant Enterobacteriaceae (CRE). Resistance to colistin can occur in Enterobacteriaceae either through adaptive chromosomal mutations that alter the bacterial outer membrane, or through plasmid-mediated horizontal gene transfer. The recently recognised global distribution of plasmid-borne colistin resistance determinants $m c r-1, m c r-2, m c r-3$ and $m c r-4$ represent a concern for infection prevention and public health, especially in the context of high CRE prevalence in hospital wards [3-5].

Recent data indicate that colistin resistance occurs more frequently among CRE than carpapenem-susceptible strains, and a recent survey conducted in Italian hospitals showed a high rate (almost $40 \%$ ) of colistin resistance among CRE, although $\mathrm{mcr}-1$ was not detected by subsequent molecular tests [6]. In this context, we would like to share the preliminary results of an ongoing retrospective and prospective study aimed at detecting the presence of $m c r-1$ and $m c r-2$ in all our colistin-resistant isolates of carbapenem-resistant Klebsiella pneumoniae. We screened our collection of 369 strains isolated between 2013 to July 2017. Most were obtained from patients treated at our tertiary hospital in Rome, and some were referred from other hospitals of the Rome area. Reduced susceptibility to colistin was recorded for $127 / 369$ (34\%), with minimal inhibitory concentrations (MICs) ranging from $2 \mathrm{mg} / \mathrm{L}$ to greater than $16 \mathrm{mg} / \mathrm{L}$. Given that some $\mathrm{mcr}$-1-positive isolates may have a colistin MIC as low as $2 \mathrm{mg} / \mathrm{L}$, we lowered our inclusion threshold to include this value. All isolates were screened by PCR for the presence of $m c r-1$ and $m c r-2$ genes as previously described $[2,3]$. The percentage of colistin resistance in our carbapenem-resistant $K$. pneumoniae strains remained relatively constant over the years at around 30\%, with the exception of 2016 when it dropped to $15 \%$, and the first half of 2017 when we recorded $44 \%$ of resistant strains. None of the isolates studied so far were found to harbour the mcr-1 or mcr-2 genes, and our research now focuses on identifying the mechanisms that caused the observed colistin resistance.

We agree with Klytmans' conclusion that improved surveillance efforts are warranted in humans, in animals and in the environment, especially given the recent discovery of $\mathrm{mcr}-3, \mathrm{mcr}-4$, and most likely further mcr resistance genes occurring in the future in Europe, and that $E$. coli strains carrying the $\mathrm{mcr}-1$ gene have been circulating in Italy at least since 2013 [7]. Our preliminary data confirm that plasmid-mediated colistin resistance in humans is still very low in the catchment area of our hospital in Italy. However, we need to urgently prevent what could become a nightmare scenario in the future, i.e. the loss of a drug that in many cases is considered a last resort.

\section{Acknowledgements}

The work on which this letter is based, was supported by "Ricerca corrente" and " $5 \mathrm{X} 1.000$ " research funds from the Italian Ministry of Health.

\section{Conflict of interest}

None declared.

Authors' contributions

Carolina Venditti and Antonino Di Caro conceived the letter and wrote the first draft. 
Carolina Venditti, Antonella Vulcano and Silvia D’Arezzo performed the laboratory work on which this letter is based.

Carla Nisii coordinated and edited the final version of the text.

All authors revised the letter and approved the final version.

\section{Reference}

1. Kluytmans J. Plasmid-encoded colistin resistance: mcr-one, two, three and counting. Euro Surveill. 2017;22(31):30588. DOI: 10.2807/1560-7917.ES.2017.22.31.30588 PMID: 28797321

2. Liu Y-Y, Wang Y, Walsh TR, Yi L-X, Zhang R, Spencer J, et al. Emergence of plasmid-mediated colistin resistance mechanism MCR-1 in animals and human beings in China: a microbiological and molecular biological study. Lancet Infect Dis. 2016;16(2):161-8. DOI: 10.1016/S1473-3099(15)00424-7 PMID: 26603172

3. Xavier BB, Lammens C, Ruhal R, Kumar-Singh S, Butaye $\mathrm{P}$, Goossens $\mathrm{H}$, et al. Identification of a novel plasmidmediated colistin-resistance gene, $\mathrm{mcr}-2$, in Escherichia coli, Belgium, June 2016. Euro Surveill. 2016;21(27):30280. DOI: 10.2807/1560-7917.ES.2016.21.27.30280 PMID: 27416987

4. Roer L, Hansen F, Stegger M, Sönksen UW, Hasman H, Hammerum AM. Novel mcr-3 variant, encoding mobile colistin resistance, in an ST131 Escherichia coli isolate from bloodstream infection, Denmark, 2014. Euro Surveill. 2017;22(31):30584. DOI: 10.2807/1560-7917. ES.2017.22.31.30584 PMID: 28797324

5. Carattoli A, Villa L, Feudi C, Curcio L, Orsini S, Luppi A, et al. Novel plasmid-mediated colistin resistance mcr-4 gene in Salmonella and Escherichia coli, Italy 2013, Spain and Belgium, 2015 to 2016. Euro Surveill. 2017;22(31):30589. DOI: 10.2807/1560-7917.ES.2017.22.31.30589 PMID: 28797329

6. Giani T, Antonelli A, Caltagirone M, Mauri C, Nicchi J, Arena $\mathrm{F}$, et al. , AMCLI-CoSA survey participants. Evolving betalactamase epidemiology in Enterobacteriaceae from Italian nationwide surveillance, October 2013: KPC-carbapenemase spreading among outpatients. Euro Surveill. 2017;22(31):30583. DOI: $10.2807 / 1560-7917 . E S .2017 .22 .31 .30583$ PMID: 28797330

7. Cannatelli A, Giani T, Antonelli A, Principe L, Luzzaro F,

Rossolini GM. First detection of the $\mathrm{mcr}^{-1}$ colistin resistance gene in Escherichia coli in Italy.Antimicrob Agents Chemother. 2016;60(5):3257-8. DOI: 10.1128/AAC.00246-16 PMID: 26976865

\section{License and copyright}

This is an open-access article distributed under the terms of the Creative Commons Attribution (CC BY 4.0) Licence. You may share and adapt the material, but must give appropriate credit to the source, provide a link to the licence, and indicate if changes were made.

This article is copyright of the authors, 2017. 